\title{
Panorama da educação em enfermagem no Brasil: graduação e pós-graduação
}

Recebido em: 14/05/2010

Aceito em: 21/03/2011
Alacoque Lorenzinni Erdmann' Josicelia Dumêt Fernandes ${ }^{2}$ Giselle Alves Teixeira ${ }^{3}$

O texto aborda a educação em enfermagem no Brasil (graduação e pós-graduação), seu panorama atual, tendências e perspectivas, objetivando esboçar sua expansão, suas tendências e perspectivas. Foram utilizados dados do Instituto Nacional de Estudos e Pesquisas Anísio Teixeira e da Coordenação de Aperfeiçoamento de Pessoal do Ensino Superior. A análise evidenciou que o sistema de educação em enfermagem deve combinar o aumento de sua capacidade de atendimento à criação de mecanismos de qualificação acadêmica, além da necessidade de um plano integrado que contemple iniciativas de redução das desigualdades regionais existentes no ensino superior. Deve, ainda, estar acoplado ao processo de avaliação como instrumento de elevação da qualidade do trabalho acadêmico.

Descritores: Pós-Graduação em Enfermagem, Educação em Enfermagem, História da Enfermagem.

\section{Overview of nursing education in Brazil: graduation and post graduation}

The text approaches the nursing education in Brazil (undergraduate and graduate), its current situation, trends and perspectives, aiming to outline its expansion, its trends and prospects. We used data from the National Institute for Research and Teixeira and the Coordination of Improvement of Higher Education. The analysis showed that the system of nursing education should combine the increase of the capacity to attend the mechanisms of academic qualification, besides the need for an integrated plan that includes initiatives to reduce regional disparities in higher education. It must also be coupled to the evaluation process as a means of raising the quality of academic work.

Descriptors: Post-graduation in Nursing, Nursing Education, Nursing History.

\section{Información general de educación en enfermería en Brasil: graduación y postgrado}

El texto analiza la formación de enfermería en Brasil (de grado y postgrado), su situación actual, tendencias y perspectivas, con el objetivo de esbozar su expansión, sus tendencias y perspectivas. Se utilizaron los datos del Instituto Nacional de Investigación y Teixeira y la Coordinación de Perfeccionamiento de la Educación Superior. El análisis mostró que el sistema de educación en enfermería debería combinarse para aumentar su capacidad para cumplir con los mecanismos de calificación académica, además de la necesidad de un plan integrado que incluye iniciativas para reducir las disparidades regionales en la educación superior. También debe ir acompañado al proceso de evaluación como un medio para elevar la calidad del trabajo académico.

Descriptores: Postgrado en Enfermería, Educación en Enfermería, Historia de la Enfermería.

\section{INTRODUÇÃO}

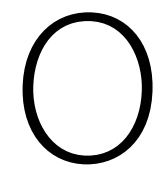

presente texto tem como objeto de estudo a educação em enfermagem no Brasil, tanto em nível de graduação quanto em pós-graduação, seu panorama atual, tendências e perspectivas. Esse objeto tem sido alvo de constante preocupação por parte da área de enfermagem, considerando-se a necessidade de fortalecimento das atividades acadêmicas voltadas para a realidade de saúde do país.

Para a abordagem da temática, partimos do entendimento de que a educação em enfermagem é construída de acordo com os limites e possibilidades de seu espaço histórico-cultural, que não é estático e está sujeito a transformações contínuas. A educação em enfermagem é apreendida, portanto, como produto de uma multiplicidade de processos sociais que resultam, historicamente, da prática da categoria e dos conjuntos sociais onde essa prática se desenvolve, modificando-se dinamicamente e ajustando-se à evolução da sociedade, de acordo com as exigências da categoria e do setor de saúde. Sob essa ótica, situamos a educação em enfermagem não como algo idealizado, abstrato, mas como parte e como produto do processo de construção da enfermagem.

Este estudo tem como objetivos esboçar a expansão da educação em enfermagem no Brasil, em nível de graduação e de pós-graduação, e expor as tendências e perspectivas de expansão dos diversos cursos de graduação e programas de pós-graduação em enfermagem no país.

Para oalcancedessesobjetivos, utilizamosdadosdisponibilizados pelo Instituto Nacional de Estudos e Pesquisas Anísio Teixeira

1 Enfermeira. Professora titular do departamento de saúde da UFSC. Pesquisadora do CNPq. Coordenadora da área de enfermagem da Capes. E-mail: alacoque@newsite.com.br. 2 Enfermeira. Professora titular da Escola de Enfermagem da UFBA. Pesquisadora do CNPq. Coordenadora adjunta da área de enfermagem da Capes. Salvador, Bahia. 3 Enfermeira. Doutoranda do programa de pós-graduação em enfermagem da UFBA. Membro do Grupo de Estudos e Pesquisas em Educação, Ética e Exercício de Enfermagem - Exerce. 
(Inep/MEC) e pela Coordenação de Aperfeiçoamento de Pessoal do Ensino Superior (Capes/MEC). Por se tratar de pesquisa com utilização de dados secundários, o estudo não foi submetido ao Comitê de Ética em Pesquisa.

Esperamos, com este estudo, contribuir para a formação de indicadores para uma política nacional de formação e capacitação de enfermeiras(os) adequada às exigências do desenvolvimento científico, tecnológico e inovador da área.

\section{A EXPANSÃO DA EDUCAÇÃO EM ENFERMAGEM NO BRASIL}

$A$ área da educação em enfermagem no Brasil, ao longo de sua existência, vem passando por transformações frente às exigências de seu papel na formação de recursos humanos com perfil adequado às necessidades de saúde da população eà legitimidade de seu papel na produção de conhecimentos inovadores e de utilidade para a sociedade.

Essas exigências se configuram num triplo desafio. Primeiro, manter um padrão de qualidade compatível com as exigências do mundo contemporâneo e com o desenvolvimento científico, tecnológico e inovador da área, incorporando os avanços pedagógicos contemporâneos. Segundo, propiciar aos alunos a capacidade de aprender, de trabalhar em equipe, de comunicarse, de ter agilidade frente às situações adversas e de ter capacidade propositiva. Terceiro, desenvolver atividades relevantes, com características interdisciplinares, capazes de contribuir para a solução de problemas nacionais e para a formação de indivíduos criativos, críticos, empreendedores e, sobretudo, cidadãos comprometidos com a ética da causa pública ${ }^{(1)}$.

Esses desafios emergem paralelamente às políticas públicas de educação, onde se observa uma expressiva expansão da educação superior, através do aumento do número de Instituições de Ensino Superior (IES) e, consequentemente, de cursos e vagas ofertadas, especialmente a partir da segunda metade do século 20. Essa política expansionista vem se apresentando numa relação direta com o desenvolvimento econômico e social do país, considerando que o Brasil apresenta baixa proporção de alunos, na faixa etária relevante, matriculados no ensino superior, não só em relação aos países mais desenvolvidos, mas também em relação aos países vizinhos na América Latina ${ }^{(2)}$.

Por outro lado, o desenvolvimento econômico, tecnológico, político, social e cultural do país apontava, à época, para a necessidade do aumento do número de brasileiros com um nível de instrução mais elevado.

A expansão de cursos de enfermagem teve início paralelamente aos demais cursos de ensino superior, a partir de 1968, com a lei 5540 - Lei da Reforma do Ensino Superior, que também institucionalizou a pós-graduação em dois níveis - mestrado e doutorado. Seu objetivo consistia em suprir o ensino de graduação em pessoal qualificado, de estimular estudos e pesquisas, além de atender às exigências de um mercado de trabalho que, baseado na sofisticação tecnológica do desenvolvimento industrial, requeria um número crescente de profissionais qualificados ${ }^{(3)}$.

Tal panorama expressa o processo que resultou na aprovação da Lei de Diretrizes e Bases da Educação Nacional $(\text { LDB })^{(4)}$, que viabilizou a ampliação quantitativa de IES e de aumento de vagas.
Ademais, essa lei sinalizou a possibilidade de mudanças, pela introdução de opções de ordem organizacional, curricular e de autonomia no panorama do setor educacional.

A LDB, viabilizando a criação de cursos, possibilitou a privatização do ensino, com a autonomia dada às instituições de ensino superior e a flexibilização dos currículos, além de direcionar a construção das diretrizes curriculares nacionais para os cursos de graduação ${ }^{(5)}$.

Nesse contexto, o Plano Nacional de Educação(6) apresentou uma política de Estado para o decênio 2001-2010, estabelecendo, entre outras metas, uma ampliação da oferta de vagas em cursos superiores, compatível com $30 \%$ dos jovens entre 18 e 24 anos.

A partir de então, observa-se uma expansão desenfreada de novas instituições e cursos na educação superior, evidenciando que, na área da saúde, essa expansão não ocorreu para atender às necessidades de saúde da população, mas sim para atender às demandas do desenvolvimento econômico, tecnológico, político, social e cultural do país ${ }^{(7)}$.

Esse processo expansionista, por sua vez, vem exigir maior formação do corpo docente, respondendo às necessidades de especialização para atender à área tecnológica e a uma demanda maior do processo produtivo, além de enfrentar as influências das universidades estrangeiras. Assim, além de expandir o número de cursos e de vagas, entre outras coisas, a reforma universitária, Lei 5540, em seu art. 17b, institucionalizou a pós-graduação em dois níveis (mestrado e doutorado), com os objetivos de formar pessoal qualificado para suprir o ensino superior que, ao ser ampliado, necessitaria aumentar seu corpo docente, além de estimular estudos e pesquisas para o desenvolvimento do país(3).

No Brasil, a pós-graduação foi instituída pela LDB, em 1961, e aprovada pelo Conselho Federal de Educação, em 1965, através do parecer 977.

Na área da enfermagem, a pós-graduação stricto sensu teve seu início em 1972, com a criação do curso de mestrado de enfermagem da Universidade Federal do Rio de Janeiro (Escola Ana Nery). Ainda nessa década, foram implantados mais sete cursos de mestrado: quatro na Região Sudeste, dois na Região Nordeste e um na Região Sul. E, em 1981, teve início o primeiro curso de doutorado: o Programa Interunidades de Doutorado em Enfermagem, parceria da Escola de Enfermagem da Universidade de São Paulo com a Escola de Enfermagem de Ribeirão Preto, da Universidade de São Paulo. A partir de então, a pós-graduação em enfermagem foi se expandindo em número de programas e cursos, mas num ritmo bem abaixo da expansão dos cursos de graduação.

\section{TENDÊNCIAS E PERSPECTIVAS DA EXPANSÃO DA GRADUAÇÃO E PÓS-GRADUAÇÃO EM ENFERMAGEM}

A origem do ensino superior em enfermagem remonta aos anos 1920, período marcado por grandes mudanças geradas pelo processo de urbanização e industrialização do país. De 1923 a 1947, foram criados, no Brasil, 16 cursos de enfermagem. No período de 1947 a 1964, foram criados mais 23, ou seja, um crescimento de 43,75\% em 17 anos. Esse crescimento foi mais acentuado a partir do fim da década de 1960, quando ocorreu a expansão do ensino de enfermagem no país. Assim, se em 1974 havia 41 cursos de 
graduação em enfermagem, no período de 1975 a 1977 foram implantados mais $22^{(3,7)}$.

De acordo com o MEC/Inep/Deas, em 1964 existiam 39 cursos e, em 1991, o número subiu para 106 cursos, ou seja, uma expansão de 171\%. Em 2004, esse total saltou para 415, o que significa um incremento de 291,5\%. Essa expansão foi mais acentuada a partir da LDB(4), de 1996, pois, de 1991 até 1996, foram criados apenas cinco cursos, ou seja, um incremento de apenas 4,71\%, enquanto que, de 1991 a 2010, essa expansão foi de $645,28 \%$. O gráfico 1 mostra o crescimento.

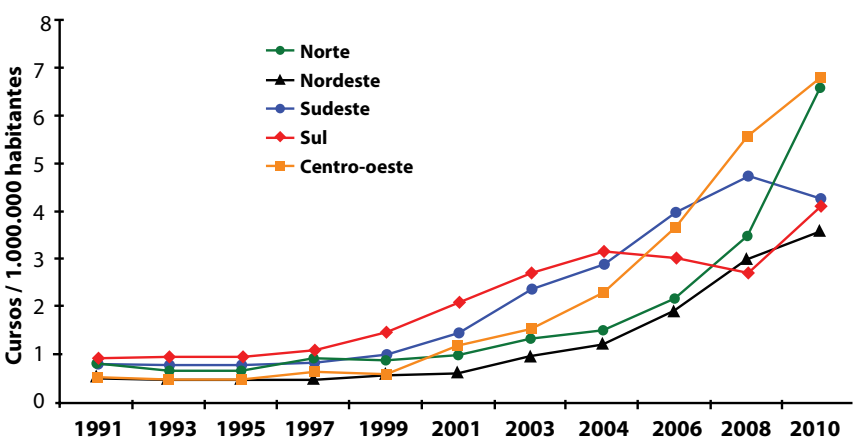

Gráfico 1 - Números de cursos de graduação em enfermagem por 1 milhão de habitantes por região geográfica, 1991-2010

Fonte MEC/Inep/Deas

Essa expansão ocorreu, predominantemente, na Região Sudeste, que absorve $43 \%$ do total de cursos de graduação em enfermagem do país, seguida da Região Nordeste, que passou a dispor de 24,12\%, Região Sul, com 14,61\%, Centro-Oeste, com 10,96\%, e Região Norte, que abriga apenas 7,3\% dos cursos.

Devido ao aumento do número de cursos na Região CentroOeste - de cinco, em 1991, para 65, em 2010 -, ela apresentou maior participação proporcional de crescimento de cursos.

Como ilustrado no gráfico 2, a expansão de cursos não vem acontecendo de maneira uniforme, existindo um forte desequilíbrio regional.

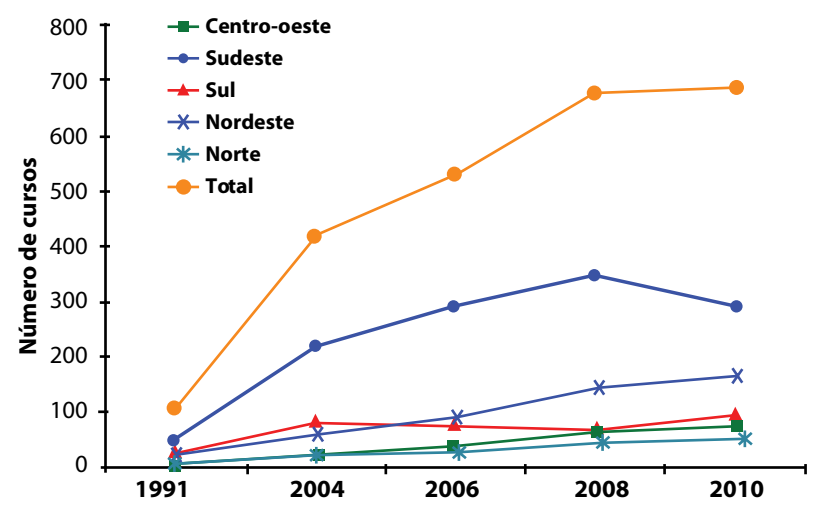

Gráfico 2 - Oferta de cursos de graduação em enfermagem por região geográfica, 1991-2010 Fonte MEC/Inep/Deas

Vale destacar, ainda, que a desigualdade na oferta de cursos se faz presente não apenas entre as regiões, mas também no interior das mesmas, ou seja, dos $43 \%$ de cursos em funcionamento na Região Sudeste, $36,73 \%$ estão no estado de São Paulo. Por outro lado, observa-se uma enorme concentração de investimentos públicos onde a capacidade instalada de recursos humanos qualificados e de infraestrutura preexistentes já é consideravelmente elevada. Onde existe o maior PIB ou maior número de cursos, o valor dos investimentos absolutos tem sido proporcionalmente maior ${ }^{(8)}$.

Observa-se, também, um desequilíbrio na distribuição dos cursos por categoria administrativa. Os dados apontam ainda para uma predominância numérica da rede privada, que engloba $85,97 \%$ dos estabelecimentos, enquanto o setor público é responsável por apenas $14,03 \%$ das instituições. As instituições privadas prevalecem numericamente em todas as regiões do país, conforme ilustrado no gráfico 3.

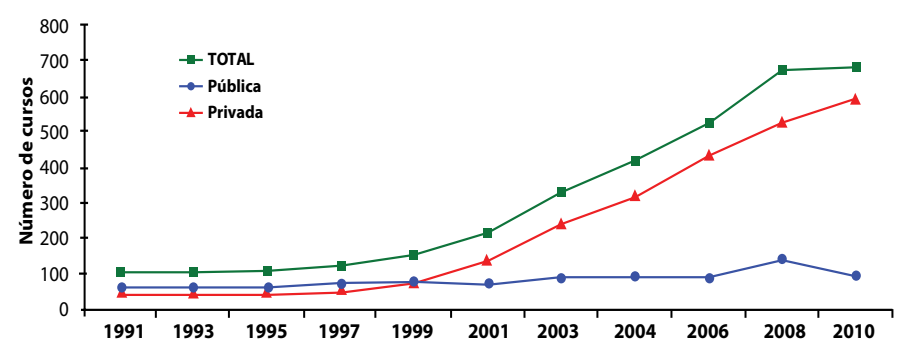

Gráfico 3 - Oferta de cursos de graduação em enfermagem por categoria administrativa, 1991-2010 Fonte MEC/Inep/Deas

Ao lado da expansão da graduação, desenvolveu-se no país, nos últimos 30 anos, um vigoroso sistema de pós-graduação. $\mathrm{Na}$ área de enfermagem, contudo, o crescimento da pósgraduação stricto sensu não ocorreu na mesma proporção que na graduação. Assim é que, se em 1974 o país contava com apenas dois cursos de mestrado em enfermagem, em 1998, passou a 14. Em 2010, por ocasião da Avaliação Trienal 20072009, a área de enfermagem contava com 41 programas de pós-graduação e 61 cursos credenciados pela Capes, sendo 20 doutorados, 38 mestrados acadêmicos e três mestrados profissionais $^{(10,11)}$. Esses dados estão ilustrados no gráfico 4.

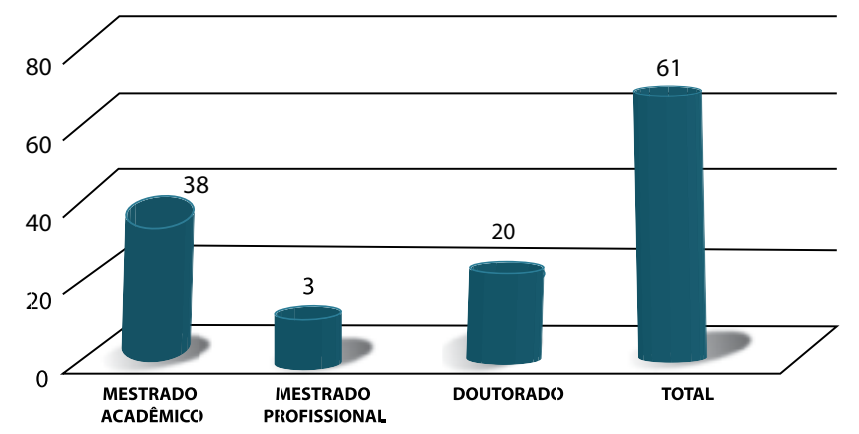

Gráfico 4 - Cursos oferecidos pelos programas de pós-graduação em enfermagem - 2010 Fonte Capes/MEC

Esses programas, após avaliação da Capes, alcançaram os seguintes conceitos/notas: conceito 3: 14 programas; conceito 4: 14 programas; conceito 5: nove programas; e conceito 6: quatro programas, conforme ilustrado no gráfico $5^{(10,11)}$. 


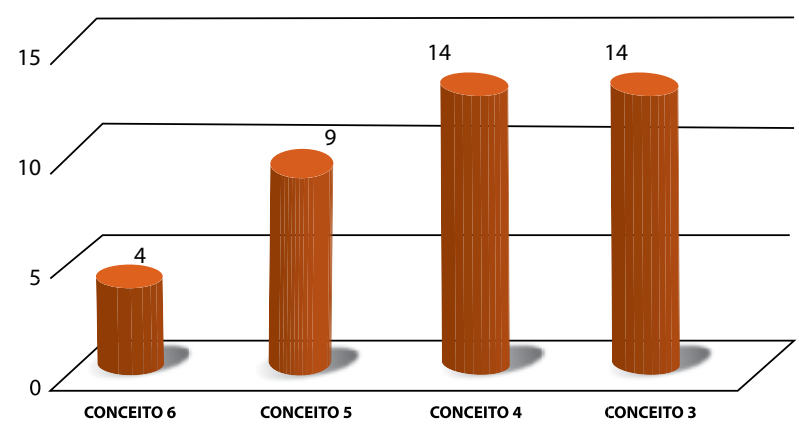

Gráfico 5 - Conceito Capes de programas de pós-graduação em enfermagem - 2010 Fonte Capes/MEC

Comparando a expansão dos dois níveis de ensino (graduação e pós-graduação) na área de enfermagem, observamos um acentuado desequilíbrio, conforme ilustrado nos gráficos 6 e 7 .

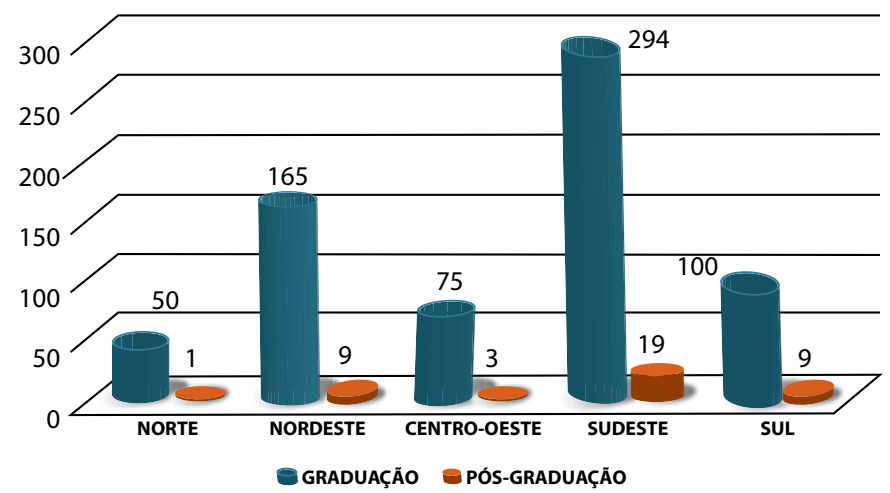

Gráfico 6 - Cursos de graduação X pós-graduação em enfermagem por região geográfica - 2010 Fonte Capes/MEC

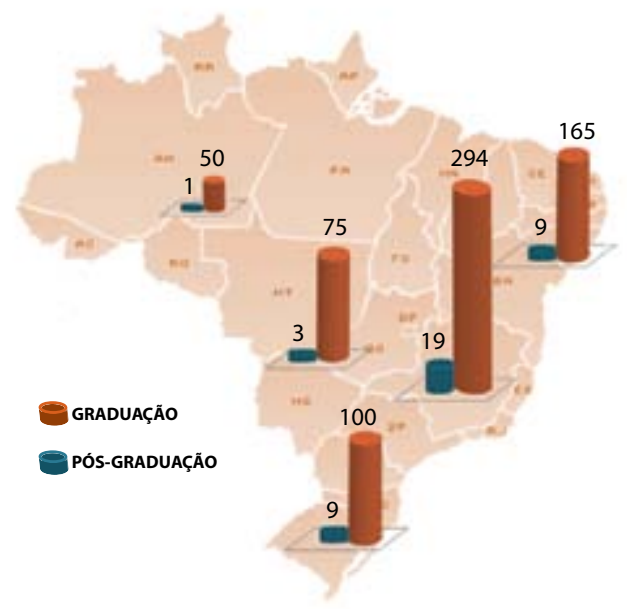

Gráfico 7 - Graduação X pós-graduação Fonte MEC/Inep

A área de enfermagem da Capes, após a Avaliação Trienal 20072009 e com a aprovação de novas propostas de criação de cursos novos - APCN-2010, finalizou o ano de 2010 com um total de 48 programas, totalizando 72 cursos, assim distribuídos: 42 MA, 23 DA e 7 MP. Desses 48 programas, 21 estão classificados com nota 3, 14 com nota 4, nove com nota 5 e quatro com nota 6.
A expansão de cursos de graduação e o consequente aumento de oferta de vagas vêm exigindo novos saberes que, por sua vez, exigem novos perfis para os profissionais, o que implica em mudanças na natureza e no processo de trabalho, além de indicar a necessidade de maior embasamento educacional dos trabalhadores, com ênfase não apenas no conhecimento técnico-científico, mas também no ético, no social, no político e no cultural, como requisitos e atributos de qualificação profissional(7).

Nesse panorama, foi aprovada, em 2004, a lei no $10861^{(9)}$, que criou o Sistema Nacional de Avaliação da Educação Superior Sinaes, tendo a qualidade da educação como meta a ser buscada através da avaliação como instrumento de política educacional.

A avaliação de cursos e de instituições aparece, destarte, como um processo necessariamente contínuo, passando a ser peça fundamental na garantia da qualidade da formação/capacitação.

Na graduação, o processo avaliativo é coordenado pela Comissão Nacional de Avaliação da Educação Superior (Conaes), sendo que a operacionalização é de responsabilidade do Inep. Na pós-graduação, esse processo é dirigido pela Coordenação da Área de Enfermagem da Capes, sendo que a operacionalização é de responsabilidade da diretoria de avaliação da Capes.

$\mathrm{Na}$ graduação, esse processo é feito através da "avaliação institucional", "avaliação de cursos" e do Exame Nacional de Desempenho do Aluno (Enade). A avaliação institucional envolve duas etapas: autoavaliação coordenada pela Comissão Própria de Avaliação (CPA) e avaliação externa realizada por comissões designadas pelo Inep/MEC, segundo diretrizes estabelecidas pela Conaes. Esse processo tem função de regulação por meio de atos administrativos para: credenciamento/recredenciamento, autorização de curso, reconhecimento de curso/renovação, descredenciamento, desativação de curso. O procedimento que envolve esse processo é realizado através da avaliação pedagógica e as instalações físicas com ênfase na biblioteca e nos laboratórios.

Na pós-graduação, o sistema de avaliação desenvolvido pela Capes envolve dois processos conduzidos por comissões de consultores oriundos de diferentes instituições do país, cumprindo papel de fundamental importância para o desenvolvimento da pósgraduação e da pesquisa científica e tecnológica no Brasil: a avaliação dos programas de pós-graduação e a avaliação das propostas de cursos novos de pós-graduação. A primeira é feita através do acompanhamento anual e da avaliação trienal do desempenho dos programas que fundamentam a deliberação do CNE/MEC sobre quais cursos vão obter a renovação de "reconhecimento", a vigorar no triênio subsequente. A avaliação das Propostas de Cursos Novos verifica o padrão de qualidade dessas propostas e se elas atendem ao requerido por esse nível de formação, fundamentando a deliberação doCNE/MEC sobreoreconhecimento detaiscursose suaincorporação ao Sistema Nacional de Pós-Graduação. Ambos os processos estão fundamentados num mesmo conjunto de princípios, diretrizes e normas, cujos procedimentos são realizados por consultores de diferentes instituições das diversas regiões do país.

\section{CONSIDERAÇÕES FINAIS}

Da análise realizada, percebemos que o sistema de educação em enfermagem, tanto em nível de graduação quanto em 
pós-graduação, ocupa uma posição fundamental no processo de modernização e desenvolvimento da atenção à saúde da população. Tem a função de capacitar e qualificar profissionais para atender às diversas e cada vez mais complexas demandas do setor de saúde e ao alcance de metas para o incremento da construção de conhecimentos relevantes e inovadores.

Percebemos, também, que a graduação se expandiu desordenadamente, em resposta às pressões da demanda por ensino superior e de grupos interessados em adquirir e/ ou acumular um capital escolar, além da penetração do setor privado, ao contrário da pós-graduação, que cresceu de forma tímida e com maior absorção pelo setor público. Vale destacar, contudo, que a expansão tanto da graduação quanto da pósgraduação evidenciam, ainda, um forte desequilíbrio regional.

A graduação cresceu em número de cursos, de alunos e professores, mas não observamos esforços contínuos para um adequado planejamento de seu crescimento, tampouco uma política de fomento para a melhoria acadêmica no que diz respeito à articulação da tríade ensino/pesquisa/extensão. A pós-graduação, ao contrário, cresceu de forma mais planejada e orientada, com planos nacionais de pós-graduação bem definidos para sua expansão. Ademais, teve a seu lado os órgãos de fomento nacionais e internacionais, que investiram de forma sistemática em seu fortalecimento. Sua estrutura acadêmica acoplou o ensino à pesquisa, estabelecendo disciplinas articuladas com as respectivas linhas de pesquisa dos programas, o que tem permitido um forte crescimento da produção científica, que, colaborando na renovação do saber na área, contribui para a introdução de novas questões para investigação. Vale destacar, ainda, sua articulação com centros relevantes da produção científica internacional.

Aspolíticaspúblicasquefundamentaramaexpansãodaeducação partiram da premissa de que a melhora da pós-graduação traria o aperfeiçoamento automático da graduação ${ }^{(7)}$. A pós-graduação, certamente, contribuiu para melhorar a titulação dos docentes que atuam no ensino superior. Mas o investimento feito nesse nível de ensino não parece ter produzido um efeito de impacto na melhoria do ensino de graduação nas diversas instituições espalhadas pelo país, apesar da criação de uma série de programas para aperfeiçoar a graduação e articulá-la melhor com a pós-graduação, tais como os Programas Institucionais de Bolsas de Iniciação Científica, patrocinados pelo CNPq, além do Programa de Educação Tutorial - PET. Esses permitem a integração de estudantes da graduação com a pós-graduação, contribuindo para elevar a qualidade da formação acadêmica dos alunos de graduação.

Vale destacar, também, que a constituição e a consolidação de grupos de pesquisa tem sido evidenciada como condição indispensável para o fortalecimento da formação/capacitação de profissionais, que deve refletir na produção de conhecimentos científicos, tecnológicos e inovadores das publicações em periódicos de impacto e em maior número de recursos humanos qualificados. É, portanto, desejável que o planejamento da pesquisa e da pósgraduação seja pensado conjuntamente com a graduação.

A expansão do ensino superior deve, ainda, combinar o aumento da capacidade de atendimento do sistema à criação de mecanismos reais que qualifiquem academicamente $o$ sistema como um todo, além de responder à necessidade de um plano integrado que contemple iniciativas de redução das desigualdades regionais existentes no ensino superior. Deve, ainda, estar acoplada ao processo de avaliação.

A reflexão sobre a educação em enfermagem, tanto em nível de graduação quanto de pós-graduação, deve estimular os cursos a se perceberem nesse processo e a reverem suas decisões pedagógicas à luz do novo paradigma da formação/capacitação do profissional de saúde, sustentado no modelo de atenção à saúde, preconizado pelo SUS e centrado na ciência, tecnologia e inovação em enfermagem.

\section{Referências}

1. Amâncio Filho A. Dilemas e desafios da formação profissional em saúde.

Interface Comun Saúde Educ. 2004;8(15):375-80.

2. Zarur GCL. O discurso liberal e a política de expansão da educação superior

no Brasil [lnternet]. [citado em 2010 Abr 15]. Disponível em: http://www.

georgezarur.com.br/pagina.php/63.

3. Fernandes JD. Expansão do ensino de enfermagem no Brasil [tese]. Salvador:

Escola de Enfermagem da Universidade Federal da Bahia; 1988.

4. Ministério da Educaçăo (BR). Lei no 9394 de 20 de dezembro de 1996 :

estabelece as diretrizes e bases da educação nacional. Brasília: Diário Oficial da

União; 1996. Dez 23;34(248). Seção 1:27.833-41.

5. Fernandes JD. A trajetória do ensino de graduação em enfermagem no Brasil.

In: Teixeira E, Vale EG, Fernandes JD, Sordi MRL, organizadores. Brasil. Ministério

da Educação. Conselho Nacional de Educação. Câmara de Educação Superior.

Resolução CNE/CES n॰ 3 de 7/11/2001: Diretrizes Curriculares Nacionais do Curso

de Graduação em Enfermagem. Brasilia (DF); 2001.

6. Ministério da Educação (BR). Lei no 10172/2001: estabelece o Plano Nacional

de Educação (PNE). Brasilia: Congresso Nacional; 2001.
7. Teixeira E, Vale EG, Fernandes JD, DE Sordi MRL. Trajetória e tendências dos

cursos de enfermagem no Brasil. Rev Bras Enferm. 2006;59(4):479-87.

8. Vieira ALS. Tendências do sistema educativo no Brasil: medicina, enfermagem

e odontologia. In: Observatório de Recursos Humanos em Saúde no Brasil:

estudos e análises. Brasilia: Ministério da Saúde; 2004.

9. Lei $n^{\circ} 10861$, de 14 de abril de 2004. Regulamenta os procedimentos de

avaliação do Sistema Nacional de Avaliação da Educação Superior. Brasilia: Diário

Oficial da Uniăo; 2004.

10. Ministério da Educação (BR). Coordenação de Aperfeiçoamento de

Pessoal de Nível Superior. Documento da Área de Enfermagem

[Internet]. [citado em 2010 Jul 10]. Disponível em:

http://www.capes.gov.br/images/stories/download/avaliacao/

ENFERMAGEM_22jun10b.pdf.

11. Relatório de Avaliação da Área da Enfermagem [Internet].

[citado em 2010 Jul 10]. Disponível em: http://trienal.capes.gov.br/

wp-content/uploads/2010/12/ENFERMAGEM-RELATÓRIO-DE-AVALIAÇÃO-

FINAL-dez10.pdf. 